\section{Strain and temperature characterisation of sensing head based on suspended-core fibre in Sagnac interferometer}

O. Frazão, J.M. Baptista, J.L. Santos, J. Kobelke and K. Schuster

The study of the strain and temperature characteristics of a sensing head based on a four-hole suspended-core fibre in a Sagnac interferometric configuration is reported. It is shown that, for the case of using an uncoated suspended-core fibre, a relatively large strain sensitivity is obtained $(\simeq 1.94 \mathrm{pm} / \mu \varepsilon)$, while the temperature sensitivity is small $\left(\simeq 0.29 \mathrm{pm} /{ }^{\circ} \mathrm{C}\right)$, pointing to a temperature-independent strain sensor. When the fibre is coated, the strain sensitivity remains essentially the same, while the temperature sensitivity becomes much larger and with a value that changes with the localisation of the temperature variation range.

Introduction: Since the first publication in 1996 on photonic crystal fibres (PCF) by Knight et al. [1], the optical fibre community has been studying the optical properties, fabrication processes and applications of this new class of fibres. A commonly accepted classification of PCF divides the fibres into two main groups: index-guiding PCF and photonic bandgap PCF. The index-guiding PCF basic structure is a solid core surrounded by a microstructured cladding. Owing to the presence of air holes, the effective refractive index of the cladding is below that of the core and the light is guided along the core by the principle of total internal reflection. Several authors have presented different geometries for this type of fibre and one of them is the suspended-core structure, where relatively large holes surround the fibre core that looks suspended along the fibre axis by small width silica walls. It was proposed initially by Monro et al. [2], and recently Webb et al. [3] demonstrated a simple technique for fabrication of these structures.

The silica suspended-core fibre has been used for gas sensing through evanescent field interaction [3, 4] . Based on the same optical phenomenon, refractometric applications have also been considered, as is illustrated in a recent work in which a fibre Bragg grating, photowritten in a suspended Ge-doped silica core, was used to measure the refractive index of liquids [5].

In this work, we propose the use of a length of four-hole suspendedcore PCF inserted in a Sagnac interferometric configuration as a sensing head for temperature-independent strain measurement. Two situations were analysed, namely the cases of using the PCF uncoated and coated.

Experimental results: Fig. 1 illustrates the experimental setup, which consists of an optical broadband source, a Sagnac interferometer containing a length of suspended-core fibre and an optical spectrum analyser (OSA) with a maximum resolution of $0.05 \mathrm{~nm}$. The optical source is an erbium-doped broadband source with a central wavelength of $1550 \mathrm{~nm}$ and a spectral bandwidth of $100 \mathrm{~nm}$. The Sagnac interferometer is formed by a $3 \mathrm{~dB}(2 \times 2)$ optical coupler with low insertion loss, an optical polarisation controller (PC) and a Hi-Bi PCF section. The suspended core was fabricated at the IPHT (Institute of Photonic Technology, Jena, Germany) and is formed by four holes with a diameter of $43.3 \mu \mathrm{m}$ (inset of Fig. 1). The core and the cladding have diameters of $\simeq 5.0$ and $\simeq 135.0 \mu \mathrm{m}$, respectively. The core fibre is slightly elliptical owing to the hole asymmetry originated during the fabrication process. This fibre was spliced at each end to a standard singlemode fibre using a conventional splicing machine. The total loss of the structure was $\simeq 11 \mathrm{~dB}$, which includes the losses of the two splices, the coupler, as well as the loss associated with the light propagation in the suspended-core fibre. The group modal birefringence of this fibre $(\beta)$ was obtained using the equation $\beta=\lambda^{2} / \Delta \lambda L$, where $\lambda$ is the operation wavelength $(1550 \mathrm{~nm})$, and $\Delta \lambda$ and $L$ are the spectral wavelength period of the interferometric fringe pattern and the length of the suspended-core fibre, respectively. Fig. 2 shows the spectral response of the interferometer. The measured spectral wavelength period was $\Delta \lambda \simeq 41.71 \mathrm{~nm}$. The length of the suspended core fibre was $\simeq 1.2 \mathrm{~m}$. Using these parameters it was found that $\beta \simeq$ $4.8 \times 10^{-5}$.

The characterisation of the sensing head when subjected to physical parameter (strain or temperature) variations was obtained by analysing the spectral variation of the first interferometer's notch. Fig. 3 shows the strain response of the structure, from which a linear response is obtained with a slope of $\simeq 1.94 \mathrm{pm} / \mu \varepsilon$ (independent of the fibre to be coated or uncoated). This value indicates that the birefringence of the four-hole suspended-core fibre increases with the increase of strain by an amount substantially larger than the one present in conventional polarisation-maintaining $(0.23 \mathrm{pm} / \mu \varepsilon$; Dong et al., [6]), certainly a consequence of its air-filled large volume holes, which reduce the fibre Young modulus.

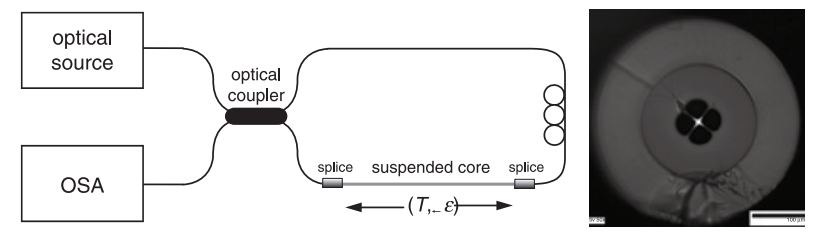

Fig. 1 Sensing head experimental setup Inset: photograph of four-hole suspended-core fibre

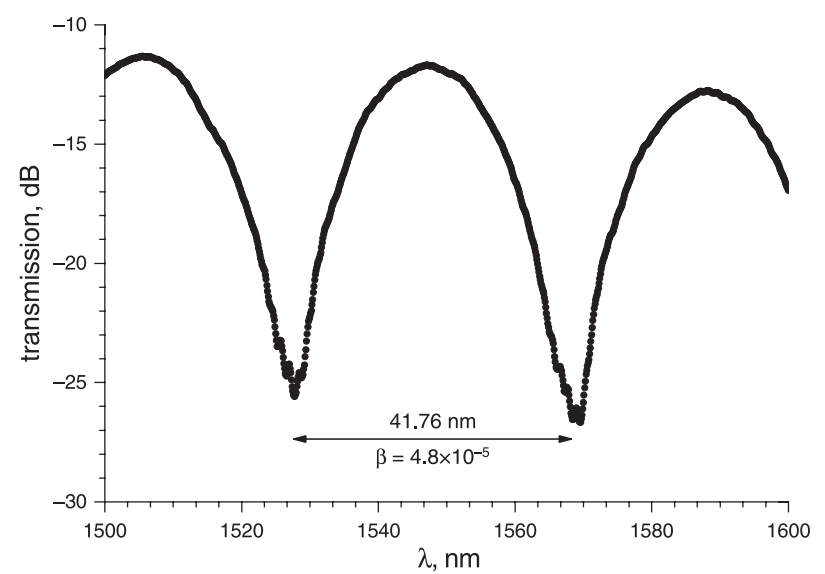

Fig. 2 Spectral transfer function of Sagnac interferometer with a length of four-hole suspended-core fibre

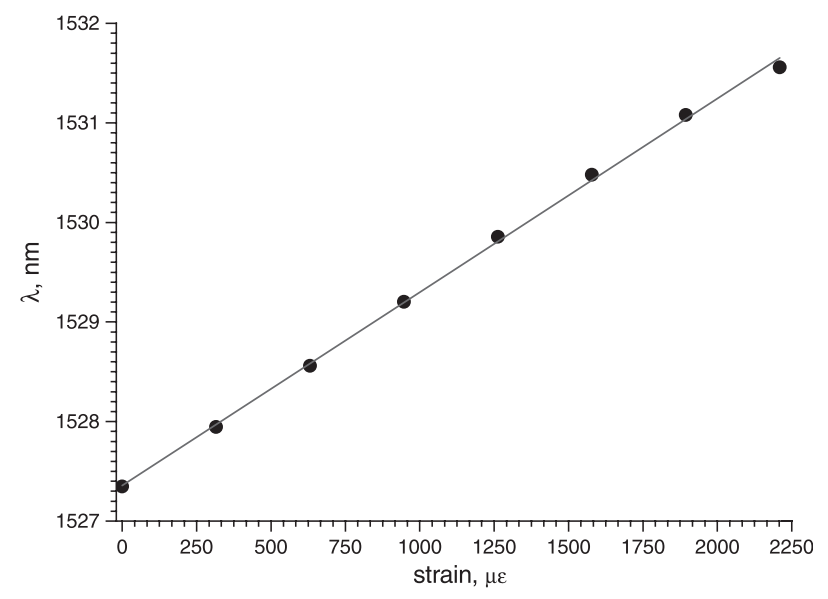

Fig. 3 Sensing head response to strain variations

The sensing head was also characterised for temperature variations. The results obtained are shown in Fig. 4. In the situation of uncoated fibre, the sensing head temperature sensitivity is small $(\simeq 0.29 \mathrm{pm} /$ ${ }^{\circ} \mathrm{C}$ ). This behaviour comes from the fact that the suspended-core fibre has a single material (pure silica), which means the absence of temperature induced internal stress that appears when doping is added to the core, often the origin of fibre birefringence variation with temperature change. On the other hand, Fig. 4 also shows an unexpected result, namely a significant shift of the interferometric channelled spectrum with temperature in the situation of coated fibre; in addition, this shift depends on the temperature region where the temperature variation occurs (slopes of $\simeq-0.10$ and $\simeq 0.07 \mathrm{~nm} /{ }^{\circ} \mathrm{C}$ for the ranges $20-40$ and $40-80^{\circ} \mathrm{C}$, respectively). The origin of this phenomenon is under investigation, but is certainly associated with the different thermal responses of the silica and acrylate materials. 


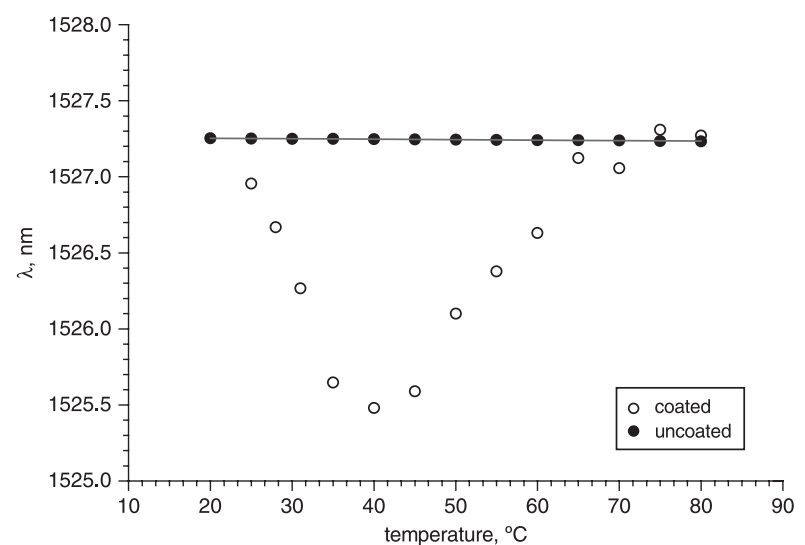

Fig. 4 Sensing head response to temperature variations

Conclusions: A sensing head structure based on a four-hole suspended-core fibre in a Sagnac interferometric configuration has been studied for strain and temperature measurements. For the case of uncoated fibres, we observed strain and temperature sensitivities of 1.94 and $0.29 \mathrm{pm} /{ }^{\circ} \mathrm{C}$, respectively, which indicates the potential for a temperature insensitive strain sensor. When the fibre is coated, the magnitude of the temperature sensitivity substantially increases, changing its signal around the temperature of $40^{\circ} \mathrm{C}$.

Acknowledgments: This work was supported by COST 299 - Optical Fibres for New Challenges Facing the Information Society. The authors thank Waclaw Urbanczyk for its contribution and support.

(C) The Institution of Engineering and Technology 2008

21 May 2008

Electronics Letters online no: 20081431

doi: 10.1049/el:20081431
O. Frazão, J.M. Baptista and J.L. Santos (INESC Porto, Rua do Campo Alegre 687, 4169-007, Porto, Portugal)

E-mail: ofrazao@inescporto.pt

J. Kobelke and K. Schuster (IPHT-Institute of Photonic Technology, D-07745 Jena, Germany)

J.M. Baptista: Also with Dept. de Matemática e Engenharias, Universidade da Madeira, Campus da Penteada, 9000-390 Funchal, Portugal

J.L. Santos: Also with Dept. de Física da Faculdade de Ciências da Universidade do Porto, Rua do Campo Alegre 687, 4169-007 Porto, Portugal

\section{References}

1 Knight, J.C., Birks, T.A., Russel, P. St., J., and Atkin, D.M.: 'All-silica single-mode optical fiber with photonic crystal cladding', Opt. Lett. 1996, 21, (19), pp. 1547-1549

2 Monro, T.M., Belardi, W., Furusawa, K., Baggett, J.C., Broderick, N.G.R., and Richardson, D.J.: 'Sensing with microstructured optical fibres', Meas. Sci. Technol., 2001, 12, pp. 854-858

3 Webb, A.S., Poletti, F., Richardson, D.J., and Sahu, J.K.: 'Suspendedcore holey fiber for evanescent-field sensing', Opt. Eng., 2007, 46 , p. 010503-1-010503-3

4 Afshar, S., Warren-Smith, S.C., and Monro, T.M.: 'Enhancement of fluorescence-based sensing using microstructured optical fibres', Opt. Express, 2007, 15, pp. 17892-17901

5 Huy, M.C.P., Laffont, G., Dewynter, V., Ferdinand, P., Roy, P., Auguste, J-L., Pagnoux, D., Blanc, W., and Dussardier, B.: 'Three-hole microstructured optical fiber for efficient fiber Bragg grating refractometer', Opt. Lett., 2007, 32, pp. 2390-2392

6 Dong, X., Tam, H.Y., and Shum, P.: 'Temperature-insensitive strain sensor with polarisation-maintaining photonics crystal fiber based on Sagnac interferometer', Appl. Phys. Lett., 2007, 90, p. 151113$1-151113-3$ 